\title{
A survey on modular vector fields and CY modular forms

$$
\text { attached to Dwork family }
$$

Uma revisão de campos vetoriais modulares e formas modulares de $C Y$ decorrentes da família de Dwork

\author{
YOUNES NIKDELAN ${ }^{a}$ b
}

\begin{abstract}
Resumo
Este artigo tem como objetivo fornecer uma revisão geral dos trabalhos do autor sobre campos vetoriais modulares e formas modulares de Calabi-Yau (CY) decorrentes da família de Dwork. Para qualquer número inteiro positivo $n$, é introduzido um espaço modui $\mathrm{T}:=\mathrm{T}_{n}$ de variedades enriquecidas de $\mathrm{CY}$ de dimensão $n$ provenientes da família de Dwork. Observa-se que existe um único campo vetorial $\mathrm{D}$ em $\mathrm{T}$, conhecido como campo vetorial modular, cujos componentes de uma solução podem ser expressos como $q$-expansões (série de Fourier) com coeficientes inteiros. Chamamos essas $q$-expansões de formas modulares de CY e verifica-se que o espaço gerado por elas possui uma estrutura canônica de $\mathfrak{s l}_{2}(\mathbb{C})$-módulo que lhe confere uma estrutura algébrica de Rankin-Cohen. Todos esses conceitos são explicitamente estabelecidos para $n=1,2,3,4$.
\end{abstract}

Palavras-chave: Campo vetorial modular, forma modular de Calabi-Yau, conexão de Gauss-Manin disfarçada.

\begin{abstract}
This article aims to give a survay of the works of the author on modular vector fields and Calabi-Yau (CY) modular forms attached to the Dwork family and avoid technical details. For any positive integer $n$, it is introduced a moduli space $\mathrm{T}:=\mathrm{T}_{n}$ of enhanced CY $n$-folds arising from the Dwork family. It is observed that there exists a unique vector field $\mathrm{D}$ in $\mathrm{T}$, known as modular vector field, whose solution components can be expressed as $q$-expansions

${ }^{a}$ Max Planck Institute for Mthematics (MPIM), Vivatsgasse 7, 53111, Bonn, Germany;

${ }^{b}$ Departamento de Análise Matemática, Instituto de Matemática e Estatística (IME), Universidade do Estado do Rio de Janeiro (UERJ), Rua São Francisco Xavier, 524, Rio de Janeiro, Brazil; ORCID: https://orcid.org/0000-0002-2479-7697 E-mail: younes.nikdelan@ime.uerj.br
\end{abstract}


(Fourier series) with integer coefficients. We call these $q$-expansions CY modular forms and it is verified that the space generated by them has a canonical $\mathfrak{s l}_{2}(\mathbb{C})$-module structure which provides it with a Rankin-Cohen algebraic structure. All these concepts are explicitly established for $n=1,2,3,4$.

Keywords: Modular vector field, Calabi-Yau modular form, Gauss-Manin conection in disguise.

\section{MSC2010: 11F11, 32M25, 34M45, 14J32, 14J15, $14 \mathrm{~N} 35$.}

\section{Introduction}

Since introducing Calabi-Yau varieties, a vast number of works in mathematics and theoretical physics have been dedicated to the study of related differential equations. The solutions of these differential equations, or system of differential equations, provide us with innumerous infinite series or $q$-expansions (Fourier series) with integer coefficients which are generating functions of certain quantities. In lower dimensions $n=1$ and $n=2$ which are related to the elliptic curves and K3 surfaces, usually these q-expansions are (quasi-)modular forms, however, in higher dimensions we can not relate them with classical quasi-modular forms. Hossein Movasati by using an algebraic method in a geometric framework, calling Gauss-Manin connection in disguise (GMCD), introduced in a more systematic way a finite number of certain $q$-expansions arising from a family of CY varieties which conjecturally can generate all other $q$-expansions emerged from the same family. He called these finite number of $q$-expansions as $C Y$ modular forms. Indeed, CY modular forms are solution components of a unique canonical vector field, calling modular vector field, in a moduli space of the considered family of CY varieties enhanced with a certain basis of the middle de Rham cohomology space. To understand better the GMCD one can start reading the paper [5] which applies the method to the families of elliptic curves, and then continue with the paper [6] or the book [7]. The author in a joint work with Movasati [8] applied GMCD to a family of CY $n$-folds, $n \in \mathbb{N}$, arising from the Dwork family, and then pushed the studies forward in subsequent papers $[9,10,11]$. The present article gives a survey of $[8,9,10,11]$ and states explicitly the essential ingredients and objects in dimensions $n=1,2,3,4$.

The present article is prepared as follows. In Section 2 we first construct a moduli space arising from the Dwork family, and then establish and discuss the main results of $[8,9,10]$ for any dimension $n$. We state explicitly the modular vector field, the associated $\mathfrak{s l}_{2}(\mathbb{C})$-module structure, solution components and some other related 
facts in dimensions $n=1,2,3,4$, respectively, in Section 3, Section 4, Section 5, Section 6.

\section{GMCD, $\mathfrak{s l}_{2}(\mathbb{C})$-mdodule structure, $\mathrm{CY}$ modular forms and algebraic RC structure}

For any positive integer $n$, similarly to mirror quintic family, we construct a oneparameter family $X:=X_{z}, z \in \mathbb{P}^{1} \backslash\{0,1, \infty\}$, of Calabi-Yau $n$-folds arising from the Dwork family:

$$
W_{z}:=\left\{z x_{0}^{n+2}+x_{1}^{n+2}+x_{2}^{n+2}+\cdots+x_{n+1}^{n+2}-(n+2) x_{0} x_{1} x_{2} \cdots x_{n+1}=0\right\} \subset \mathbb{P}^{n+1},
$$

and then obtain the moduli space $\mathrm{T}:=\mathrm{T}_{n}$ of the enhanced pairs $\left(X,\left[\alpha_{1}, \alpha_{2}, \ldots, \alpha_{n+1}\right]\right)$, where $\left\{\alpha_{1}, \alpha_{2}, \ldots, \alpha_{n+1}\right\}$ is a basis of the $n$-th algebraic de Rham cohomology $H_{\mathrm{dR}}^{n}(X)$ satisfying some specific properties. Indeed, we find:

$$
\begin{aligned}
\mathrm{T} & =\operatorname{Spec}\left(\mathbb{C}\left[t_{1}, t_{2}, \ldots, t_{\mathrm{d}}, \frac{1}{t_{n+2}\left(t_{n+2}-t_{1}^{n+2}\right) \check{t}}\right]\right), \\
\mathscr{O}_{\mathrm{T}} & =\mathbb{C}\left[t_{1}, t_{2}, \ldots, t_{\mathrm{d}}, \frac{1}{t_{n+2}\left(t_{n+2}-t_{1}^{n+2}\right) \check{t}}\right]
\end{aligned}
$$

in which $\check{t}$ is the product of $\left[\frac{n+1}{2}\right]$ number of $t_{j}$ 's and

$$
\mathrm{d}:=\mathrm{d}_{n}=\operatorname{dim} \mathrm{T}= \begin{cases}\frac{(n+1)(n+3)}{4}+1, & \text { if } n \text { is odd; } \\ \frac{n(n+2)}{4}+1, & \text { if } n \text { is even. }\end{cases}
$$

Moreover, $\Delta=t_{n+2}\left(t_{n+2}-t_{1}^{n+2}\right)$ is the discriminant of the modified Dwork family under the transformation $z=\frac{\mathrm{t}_{n+2}}{t_{1}^{n+2}}$. We observe that (see [8, Theorem 1.1]) there exist a unique vector field $\mathrm{D}:=\mathrm{D}_{n}$ and unique regular functions $\mathrm{Y}_{j} \in \mathscr{O}_{\mathrm{T}}, j=$ $1,2, \ldots, n-2$ in $\mathrm{T}$ such that the Gauss-Manin connection of the universal family of $\mathrm{T}$ composed with the vector field $\mathrm{D}$, namely $\nabla_{\mathrm{D}}$, satisfies (no worries if you do not 
know Gauss-Manin connection, just keep reading and forget it):

$$
\nabla_{\mathrm{D}}\left(\begin{array}{c}
\alpha_{1} \\
\alpha_{2} \\
\alpha_{3} \\
\vdots \\
\alpha_{n} \\
\alpha_{n+1}
\end{array}\right)=\underbrace{\left(\begin{array}{ccccccc}
0 & 1 & 0 & 0 & \cdots & 0 & 0 \\
0 & 0 & \mathrm{Y}_{1} & 0 & \cdots & 0 & 0 \\
0 & 0 & 0 & \mathrm{Y}_{2} & \cdots & 0 & 0 \\
\vdots & \vdots & \vdots & \vdots & \ddots & \vdots & \vdots \\
0 & 0 & 0 & 0 & \ldots & \mathrm{Y}_{n-2} & 0 \\
0 & 0 & 0 & 0 & \cdots & 0 & -1 \\
0 & 0 & 0 & 0 & \cdots & 0 & 0
\end{array}\right)}_{\mathrm{Y}}\left(\begin{array}{c}
\alpha_{1} \\
\alpha_{2} \\
\alpha_{3} \\
\vdots \\
\alpha_{n} \\
\alpha_{n+1}
\end{array}\right) .
$$

We conjecture that component solutions of $\mathrm{D}$ can be expressed as $q$-expansions with integer coefficients (this is verified for $n=1,2,3,4$, which are stated in the next sections). For all $n$, we can find vector fields $\mathrm{W}:=\mathrm{W}_{n}$ and $\delta:=\delta_{n}$ in $\mathrm{T}$ which along with $\mathrm{D}$ form a copy of $\mathfrak{s l}_{2}(\mathbb{C})($ see $[9$, Theorem 1.4$])$, i.e.:

$$
[\mathrm{D}, \delta]=\mathrm{W}, \quad[\mathrm{W}, \mathrm{D}]=2 \mathrm{D}, \quad[\mathrm{W}, \delta]=-2 \delta,
$$

where $[\cdot, \cdot]$ refers to the Lie bracket of vector fields. Note that the vector fields $\mathrm{D}, \mathrm{W}, \delta$ in $[8,9,10,11]$ were denoted by $\mathrm{R}, \mathrm{H}, \mathrm{F}$, respectively. Indeed, we observe that $\mathrm{W}$ and $\delta$ are in the following forms:

$$
\begin{aligned}
& \mathrm{W}=\sum_{j=1}^{\mathrm{d}} w_{j} t_{j} \frac{\partial}{\partial t_{j}}, \text { for some } w_{j} \in \mathbb{Z}_{\geq 0}, \\
& \delta=\frac{\partial}{\partial t_{2}}, \text { if } n \neq 2 \quad\left(\delta=2 \frac{\partial}{\partial t_{2}}, \text { if } n=2\right) .
\end{aligned}
$$

We should mention that for all odd $n \geq 3$, as we will see in Section 5 , we will need to use a simple transformation to get $\delta$ as above and substitute $t_{\mathrm{d}}$ by $\tilde{t}_{\mathrm{d}}$, which, by abuse of notation, will be denoted again by $t_{\mathrm{d}}$.

If alternately, by abuse of notation, we suppose that $t_{j}$ 's are solution components of $\mathrm{D}$, then we can consider $\mathrm{D}, \mathrm{W}, \delta$ as differential operators on the $\mathbb{C}$-algebra generated by $t_{j}$ 's :

$$
\mathscr{M}:=\mathbb{C}\left[t_{1}, t_{2}, t_{3}, \ldots, t_{\mathrm{d}}, \frac{1}{t_{n+2}\left(t_{n+2}-t_{1}^{n+2}\right) \check{t}}\right]
$$

which is called the space of $C Y$ modular forms. By setting $\operatorname{deg} t_{j}:=w_{j}$, we provide 
the $\mathbb{C}$-algebra $\mathscr{M}$ with an algebraic graded structure, i.e.:

$$
\mathscr{M}=\bigoplus_{k \in \mathbb{Z}} \mathscr{M}_{k}
$$

in which $\mathscr{M}_{k}:=\{f \in \mathscr{M} \mid \operatorname{deg} f=k\}$, for all $k \in \mathbb{Z}$, is the space of CY modular forms of weight $k$. In particular, for all $n \in \mathbb{N}$, we observe that $w_{1}=1, w_{2}=$ $2, w_{n+2}=n+2$. Using the assigned degrees (weights) it turns out that $\mathrm{D}$ is a quasi-homogeneous vector field of degree 2 in $\mathrm{T}$, and consequently it is a degree 2 differential operator on $\mathscr{M}$, i.e., for all $f \in \mathscr{M}_{k}$, we get $\mathrm{D} f \in \mathscr{M}_{k+2}$. Analogously, we observe that $\mathrm{W} f=k f$, which is the operator multiplication by weight and $\delta f \in \mathscr{M}_{k-2}$ decreases the weight by 2 .

By comparing the $\mathfrak{s l}_{2}(\mathbb{C})$-module structure of the space of full quasi-modular forms with the $\mathfrak{s l}_{2}(\mathbb{C})$-module structure of the space of $\mathrm{CY}$ modular forms $\mathscr{M}$, it turns out that $t_{2}$ plays the role of the quasi-modular form $E_{2}$ (which is the weight 2 Eisenstein series). In this way, we introduce the space of $2 C Y$ modular forms $\mathscr{M}^{2}$ as follows:

$$
\mathscr{M}^{2}:=\mathbb{C}\left[t_{1}, t_{3}, \ldots, t_{\mathrm{d}}, \frac{1}{t_{n+2}\left(t_{n+2}-t_{1}^{n+2}\right) \check{t}}\right]=\bigoplus_{k \in \mathbb{Z}} \mathscr{M}^{2}{ }_{k}
$$

Indeed, we have $\mathscr{M}=\mathscr{M}^{2}\left[t_{2}\right]$. In a conceptual comparison, the space of $2 \mathrm{CY}$ modular forms $\mathscr{M}^{2}$ is equivalent to the space of full modular forms $\mathcal{M}\left(\mathrm{SL}_{2}(\mathbb{Z})\right)=$ $\mathbb{C}\left[E_{4}, E_{6}\right]$, and the space of $\mathrm{CY}$ modular forms $\mathscr{M}=\mathscr{M}^{2}\left[t_{2}\right]$ is equivalent to the space of full quasi-modular forms $\widetilde{\mathcal{M}}\left(\mathrm{SL}_{2}(\mathbb{Z})\right)=\mathcal{M}\left(\mathrm{SL}_{2}(\mathbb{Z})\right)\left[E_{2}\right]=\mathbb{C}\left[E_{2}, E_{4}, E_{6}\right]$. The reason for choosing the name " $2 \mathrm{CY}$ modular forms" is because of the order of appearance of this space in the literature. In fact the space of $1 \mathrm{CY}$ modular forms is $\mathscr{M}^{1}:=\mathbb{C}\left[t_{1}, t_{n+2}, \frac{1}{t_{n+2}\left(t_{n+2}-t_{1}^{n+2}\right)}\right]$ which has been studied by Movasati [7].

Let $f \in \mathscr{M}^{2}{ }_{k}$ be a $2 \mathrm{CY}$ modular form of weight $k$ for some $k \in \mathbb{Z}$. It is obvious that $\mathrm{D} f \in \mathscr{M}_{k+2}$, however it is not necessarily a $2 \mathrm{CY}$ modular form. Analogous to the Ramanujan-Serre derivation for full modular forms, we define the degree 2 Ramanujan-Serre-type derivation $\partial: \mathscr{M}^{2}{ }_{*} \rightarrow \mathscr{M}^{2}{ }^{*+2}$ as follows:

$$
\begin{aligned}
& \partial f=\mathrm{D} f+k t_{2} f, \quad \text { if } n \neq 2, \\
& \partial f=\mathrm{D} f+\frac{k}{2} t_{2} f, \quad \text { if } n=2 .
\end{aligned}
$$

Since $\mathrm{D}$ is a degree 2 derivation on $\mathscr{M}$, due to Zagier [12], we can provide $\mathscr{M}$ with a standard Rankin-Cohen (RC) structure by defining the $m$-th $R C$ bracket for 
CY modular forms as follows:

$$
[f, g]_{\mathrm{D}, m}:=\sum_{i+j=m}(-1)^{j}\left(\begin{array}{c}
m+k-1 \\
i
\end{array}\right)\left(\begin{array}{c}
m+l-1 \\
j
\end{array}\right) \mathrm{D}^{j} f \mathrm{D}^{i} g, \forall f \in \mathscr{M}_{k}, \forall g \in \mathscr{M}_{l},
$$

where $m \in \mathbb{Z}_{\geq 0}, k, l \in \mathbb{Z}$ and $\mathrm{D}^{j} f, \mathrm{D}^{i} g$ are respectively the $j$-th and $i$-th derivative of $f$ and $g$ with respect to the derivation D. It is evident that $[f, g]_{\mathrm{D}, m} \in \mathscr{M}_{k+l+2 m}$. Cohen proved that the RC bracket of modular forms is again a modular form. Similarly, we can observe that $\mathrm{RC}$ bracket of $2 \mathrm{CY}$ modular forms is again a $2 \mathrm{CY}$ modular form, i.e.,

$$
\forall f \in \mathscr{M}^{2}{ }_{k}, \forall g \in \mathscr{M}^{2}{ }_{l} \Longrightarrow[f, g]_{\mathrm{D}, m} \in \mathscr{M}^{2}{ }_{k+l+2 m}
$$

To prove this, we first let:

$$
\Lambda:= \begin{cases}-\frac{1}{2} \mathrm{D} t_{2}-\frac{1}{4} t_{2}^{2}, & \text { if } n=2, \\ -\mathrm{D} t_{2}-t_{2}^{2}, & \text { if } n \neq 2\end{cases}
$$

and observe that $\Lambda \in \mathscr{M}^{2}{ }_{4}$. For any $m \in \mathbb{Z}_{\geq 0}$ we define the brackets $[\cdot, \cdot]_{\partial, \Lambda, m}$ : $\mathscr{M}^{2}{ }_{k} \times \mathscr{M}^{2}{ }_{l} \rightarrow \mathscr{M}^{2}{ }_{k+l+2 m}:$

$$
[f, g]_{\partial, \Lambda, m}=\sum_{i+j=m}(-1)^{j}\left(\begin{array}{c}
m+k-1 \\
i
\end{array}\right)\left(\begin{array}{c}
m+l-1 \\
j
\end{array}\right) f_{(j)} g_{(i)}
$$

where $f \in \mathscr{M}^{2}{ }_{k}, g \in \mathscr{M}^{2}{ }_{l}$, and $f_{(j)} \in \mathscr{M}^{2}{ }_{k+2 j}, g_{(i)} \in \mathscr{M}^{2}{ }_{l+2 i}$ are defined recursively as follows

$$
f_{(j+1)}=\partial f_{(j)}+j(j+k-1) \Lambda f_{(j-1)}, g_{(i+1)}=\partial g_{(i)}+i(i+l-1) \Lambda g_{(i-1)},
$$

with initial conditions $f_{(0)}=f, g_{(0)}=g, f_{(1)}=\partial f, g_{(0)}=\partial g$. Then we obtain:

$$
[f, g]_{\mathrm{D}, m}=[f, g]_{\partial, \Lambda, m}
$$

which shows $[f, g]_{\mathrm{D}, m} \in \mathscr{M}^{2}{ }_{k+l+2 m}$. By this we provide $\left(\mathscr{M}^{2},[f, g]_{\mathrm{D}, m}\right)$ with a canonical RC algebra structure, in the sense Zagier [12].

In the subsequent sections we state $\mathrm{D}, \mathrm{W}, \delta$ and component solutions of $\mathrm{D}$ for $n=1,2,3,4$. In what follows $E_{2 j}, j=1,2,3$, are Eisenstein series defined as $E_{2 j}(q)=1+b_{j} \sum_{k=1}^{\infty} \sigma_{2 j-1}(k) q^{k}$ with $\left(b_{1}, b_{2}, b_{3}\right)=(-24,240,-504)$ and $\sigma_{j}(k)=$ $\sum_{d \mid k} d^{j}$, and $\eta(q)=q^{\frac{1}{24}} \prod_{k=1}^{\infty}\left(1-q^{k}\right)$ is the Dedekind eta function. We also consider 
$q=e^{2 \pi i \tau}$ where $\tau \in \mathbb{C}$ with $\operatorname{Im} \tau>0$.

\section{The case $n=1$}

In this case we find:

$$
\begin{aligned}
& \mathrm{D}=\left(-9\left(t_{1}^{3}-t_{3}\right)-t_{2} t_{1}\right) \frac{\partial}{\partial t_{1}}+\left(81 t_{1}\left(t_{1}^{3}-t_{3}\right)-t_{2}^{2}\right) \frac{\partial}{\partial t_{2}}+\left(-3 t_{2} t_{3}\right) \frac{\partial}{\partial t_{3}} \\
& \mathbf{W}=t_{1} \frac{\partial}{\partial t_{1}}+2 t_{2} \frac{\partial}{\partial t_{2}}+3 t_{3} \frac{\partial}{\partial t_{3}} \\
& \delta=\frac{\partial}{\partial t_{2}}
\end{aligned}
$$

In particular, vector field (3.2) implies $\operatorname{deg}\left(t_{1}\right)=1, \operatorname{deg}\left(t_{2}\right)=2$ and $\operatorname{deg}\left(t_{3}\right)=3$. We find a solution of $D$ in terms of quasi-modular forms in $\widetilde{\mathcal{M}}\left(\Gamma_{0}(3), \chi_{-3}\right)$ (actually, $t_{1}$ and $t_{3}$ are modular forms and $t_{2}$ is a quasi-modular form, see [11, §3]). Indeed, by using the transformations $\mathcal{P}_{3}=-2 t_{2}-9 t_{1}^{2}, \mathcal{Q}_{3}=9 t_{1}^{2}, \mathcal{R}_{3}=3 t_{1} t_{3}$ and $\mathcal{S}_{3}=t_{3}^{2}$, we find the following Ramanujan-type system for $\Gamma_{0}(3)$ :

$$
\left\{\begin{array}{l}
\mathcal{P}_{3}^{\prime}=\frac{1}{6}\left(\mathcal{P}_{3}^{2}-Q_{3}^{2}\right) \\
\mathcal{Q}_{3}^{\prime}=\frac{1}{3}\left(\mathcal{P}_{3} \mathcal{Q}_{3}-Q_{3}^{2}+54 \mathcal{R}_{3}\right) \\
\mathcal{R}_{3}^{\prime}=\frac{2}{3} \mathcal{P}_{3} \mathcal{R}_{3}+\frac{1}{3} Q_{3} \mathcal{R}_{3}+9 \mathcal{S}_{3} \\
\mathcal{S}_{3}^{\prime}=\mathcal{P}_{3} \mathcal{S}_{3}+\mathcal{Q}_{3} \mathcal{S}_{3}
\end{array} \quad *^{\prime}=q \frac{\partial}{\partial q}=\frac{1}{2 \pi i} \frac{d}{d \tau}\right.
$$

in which the relation $\mathcal{R}_{3}^{2}-Q_{3} \mathcal{S}_{3}=0$ holds. A particular solution of this system is given as follows (see [11, Theorem 1.2]):

$$
\left\{\begin{array}{l}
\mathcal{P}_{3}(q)=\frac{1}{4}\left(E_{2}(q)+3 E_{2}\left(q^{3}\right)\right) \\
\mathcal{Q}_{3}(q)=\frac{1}{2}\left(3 E_{2}\left(q^{3}\right)-E_{2}(q)\right) \\
\mathcal{R}_{3}(q)=\eta^{8}\left(q^{3}\right)+9 \frac{\eta^{8}\left(q^{3}\right) \eta^{3}\left(q^{9}\right)}{\eta^{3}(q)} \\
\mathcal{S}_{3}(q)=\left(\frac{\eta^{9}\left(q^{3}\right)}{\eta^{3}(q)}\right)^{2}
\end{array}\right.
$$

where $\mathcal{Q}_{3} \in \mathcal{M}_{2}\left(\Gamma_{0}(3)\right), \mathcal{R}_{3} \in \mathcal{M}_{4}\left(\Gamma_{0}(3)\right), \mathcal{S}_{3} \in \mathcal{M}_{6}\left(\Gamma_{0}(3)\right)$ and $\mathcal{P}_{3} \in \widetilde{\mathcal{M}}_{2}\left(\Gamma_{0}(3)\right)$. Moreover, if we consider

$$
\Delta_{3}:=\eta^{6}(q) \eta^{6}\left(q^{3}\right)
$$

which is a cusp form of weight 6 for $\Gamma_{0}(3)$, then $\Delta_{3}=2_{3} \mathcal{R}_{3}-27 \mathcal{S}_{3}$ and it is a factor of the discriminant of the Dwork family (which can be called modular discriminant for $\left.\Gamma_{0}(3)\right)$ satisfying:

$$
\Delta_{3}^{\prime}=\mathcal{P}_{3} \Delta_{3}
$$


If we consider $\mathcal{Q}_{3}, \mathcal{R}_{3}, \mathcal{S}_{3}$ as free parameters and let $\mathcal{J}:=\left\langle\mathcal{R}_{3}^{2}-\mathcal{Q}_{3} \mathcal{S}_{3}\right\rangle$ to be the ideal generated by $\mathcal{R}_{3}^{2}-\mathcal{Q}_{3} \mathcal{S}_{3}$ in $\mathbb{C}\left[Q_{3}, \mathcal{R}_{3}, \mathcal{S}_{3}\right]$, then:

$$
\begin{aligned}
& \mathcal{M}\left(\Gamma_{0}(3)\right) \simeq \frac{\mathbb{C}\left[\mathcal{Q}_{3}, \mathcal{R}_{3}, \mathcal{S}_{3}\right]}{\mathcal{J}}, \\
& \tilde{\mathcal{M}}\left(\Gamma_{0}(3)\right) \simeq \frac{\mathbb{C}\left[\mathcal{P}_{3}, \mathcal{Q}_{3}, \mathcal{R}_{3}, \mathcal{S}_{3}\right]}{\mathcal{J}} .
\end{aligned}
$$

\section{The case $n=2$}

In this case we find:

$$
\begin{aligned}
\mathrm{D} & =\left(t_{3}-t_{2} t_{1}\right) \frac{\partial}{\partial t_{1}}+\left(2 t_{1}^{2}-\frac{1}{2} t_{2}^{2}\right) \frac{\partial}{\partial t_{2}}+\left(8 t_{1}^{3}-2 t_{2} t_{3}\right) \frac{\partial}{\partial t_{3}}+\left(-4 t_{2} t_{4}\right) \frac{\partial}{\partial t_{4}} \\
\mathbf{W} & =2 t_{1} \frac{\partial}{\partial t_{1}}+2 t_{2} \frac{\partial}{\partial t_{2}}+4 t_{3} \frac{\partial}{\partial t_{3}}+8 t_{4} \frac{\partial}{\partial t_{4}} \\
\delta & =2 \frac{\partial}{\partial t_{2}}
\end{aligned}
$$

where the polynomial equation $t_{3}^{2}=4\left(t_{1}^{4}-t_{4}\right)$ holds among $t_{i}$ 's. From (4.2) we get $\operatorname{deg}\left(t_{1}\right)=2, \operatorname{deg}\left(t_{2}\right)=2, \operatorname{deg}\left(t_{3}\right)=4$ and $\operatorname{deg}\left(t_{4}\right)=8$. We get a solution of $\mathrm{D}$ in terms of quasi-modular forms in $\tilde{\mathcal{M}}\left(\Gamma_{0}(2)\right)$ (actually, $t_{1}$ and $t_{3}$ are modular forms and $t_{2}$ is a quasi-modular form). Indeed, by using the transformations $\mathcal{P}_{2}=20 t_{2}$, $Q_{2}=40 t_{1}$ and $\mathcal{R}_{2}=800 t_{3}$, we find the following Ramanujan-type system for $\Gamma_{0}(2)$ :

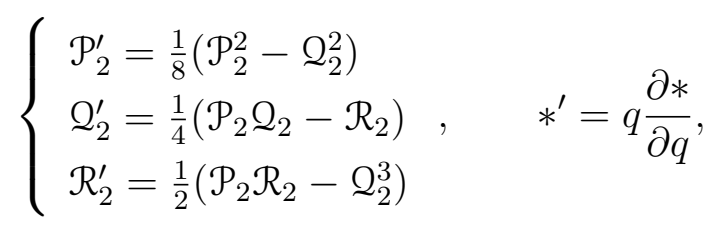

whose a particular solution is given as follows (see [11, Theorem 1.1]):

$$
\left\{\begin{array}{l}
\mathcal{P}_{2}(q)=\frac{1}{3}\left(E_{2}(q)+2 E_{2}\left(q^{2}\right)\right), \\
\mathcal{Q}_{2}(q)=2 E_{2}\left(q^{2}\right)-E_{2}(q), \\
\mathcal{R}_{2}(q)=\frac{1}{3}\left(4 E_{4}\left(q^{2}\right)-E_{4}(q)\right),
\end{array}\right.
$$

in which $Q_{2} \in \mathcal{M}_{2}\left(\Gamma_{0}(2)\right), \mathcal{R}_{2} \in \mathcal{M}_{4}\left(\Gamma_{0}(2)\right)$ and $\mathcal{P}_{2} \in \tilde{\mathcal{M}}_{2}\left(\Gamma_{0}(2)\right)$. Moreover, if we consider

$$
\Delta_{2}:=\eta^{8}(q) \eta^{8}\left(q^{2}\right)
$$

which is a cusp form of weight 8 for $\Gamma_{0}(2)$, then $\Delta_{2}=\frac{1}{256}\left(Q_{2}^{4}-\mathcal{R}_{2}^{2}\right)$ and it is a factor of the discriminant of the Dwork family (which can be called modular discriminant 
for $\left.\Gamma_{0}(2)\right)$ satisfying:

$$
\Delta_{2}^{\prime}=\mathcal{P}_{2} \Delta_{2}
$$

We also have:

$$
\begin{aligned}
& \mathcal{M}\left(\Gamma_{0}(2)\right)=\mathbb{C}\left[\mathcal{Q}_{2}, \mathcal{R}_{2}\right] \\
& \tilde{\mathcal{M}}\left(\Gamma_{0}(2)\right)=\mathbb{C}\left[\mathcal{P}_{2}, Q_{2}, \mathcal{R}_{2}\right]
\end{aligned}
$$

\section{The case $n=3$}

In this case we obtain:

$$
\begin{aligned}
\mathrm{D} & =\left(t_{3}-t_{2} t_{1}\right) \frac{\partial}{\partial t_{1}}+\left(\frac{t_{3}^{3} t_{4}}{5^{4}\left(t_{1}^{5}-t_{5}\right)}-t_{2}^{2}\right) \frac{\partial}{\partial t_{2}} \\
& +\left(\frac{t_{3}^{3} t_{6}}{5^{4}\left(t_{1}^{5}-t_{5}\right)}-3 t_{2} t_{3}\right) \frac{\partial}{\partial t_{3}}+\left(-t_{7}-t_{2} t_{4}\right) \frac{\partial}{\partial t_{4}} \\
& +\left(-5 t_{2} t_{5}\right) \frac{\partial}{\partial t_{5}}+\left(5^{5} t_{1}^{3}-2 t_{3} t_{4}-t_{2} t_{6}\right) \frac{\partial}{\partial t_{6}}+\left(-5^{4} t_{1} t_{3}-t_{2} t_{7}\right) \frac{\partial}{\partial t_{7}} \\
\mathbf{W} & =t_{1} \frac{\partial}{\partial t_{1}}+2 t_{2} \frac{\partial}{\partial t_{2}}+3 t_{3} \frac{\partial}{\partial t_{3}}+5 t_{5} \frac{\partial}{\partial t_{5}}+t_{6} \frac{\partial}{\partial t_{6}}+2 t_{7} \frac{\partial}{\partial t_{7}} \\
\delta & =\frac{\partial}{\partial t_{2}}-t_{4} \frac{\partial}{\partial t_{7}} .
\end{aligned}
$$

Hence $\operatorname{deg}\left(t_{1}\right)=1, \operatorname{deg}\left(t_{2}\right)=2, \operatorname{deg}\left(t_{3}\right)=3, \operatorname{deg}\left(t_{4}\right)=0, \operatorname{deg}\left(t_{5}\right)=5, \operatorname{deg}\left(t_{6}\right)=$ $1, \operatorname{deg}\left(t_{7}\right)=2$. We can find the $q$-expansion of a solution of $\mathrm{D}$, whose first 7 coefficients are given in Table 1.

\begin{tabular}{|c|c|c|c|c|c|c|c|}
\hline & $q^{0}$ & $q^{1}$ & $q^{2}$ & $q^{3}$ & $q^{4}$ & $q^{5}$ & $q^{6}$ \\
\hline$\frac{1}{24} t_{1}$ & $\frac{1}{120}$ & 1 & 175 & 117625 & 111784375 & 126958105626 & 160715581780591 \\
\hline$\frac{1}{10} t_{2}$ & $-\frac{1}{10}$ & 17 & 11185 & 12261425 & 16166719625 & 23478405649152 & 36191848368238417 \\
\hline$\frac{1}{10} t_{3}$ & $-\frac{1}{50}$ & 13 & 6425 & 6744325 & 8719953625 & 12525150549888 & 19171976431076873 \\
\hline$\frac{1}{125} t_{4}$ & $-\frac{1}{5}$ & 13 & 2860 & 1855775 & 1750773750 & 1981335668498 & 2502724752660128 \\
\hline$-t_{5}$ & 0 & -1 & 170 & 41475 & 32183000 & 32678171250 & 38612049889554 \\
\hline$\frac{1}{25} t_{6}$ & $-\frac{3}{5}$ & 187 & 28760 & 16677425 & 15028305250 & 16597280453022 & 20644227272244012 \\
\hline$\frac{21}{750} t_{7}$ & $\frac{1}{30}$ & 3 & 930 & 566375 & 526770000 & 592132503858 & 745012928951258 \\
\hline
\end{tabular}

Table 1: Coefficients of $q^{k}, 0 \leq k \leq 6$, in the q-expansion of a solution of $\mathrm{D}=\mathrm{D}_{3}$.

Using this solution we obtain:

$$
\mathrm{Y}_{1}=\frac{t_{3}^{3}}{5^{4}\left(t_{1}^{5}-t_{5}\right)}=5+2875 \frac{q}{1-q}+609250 \times 2^{3} \frac{q^{2}}{1-q^{2}}+\ldots
$$

which is the Yukawa coupling given in [1].

As we can see, in this case $\delta$ is different from the form claimed in (2.6) (this 
happens in all odd cases $\geq 3$ ). We can solve this problem using the transformation:

$$
\tilde{t}_{7}:=t_{7}+t_{2} t_{4}
$$

from which we obtain:

$$
\begin{aligned}
& \mathrm{D}\left(\tilde{t}_{7}\right)=-5^{4} t_{1} t_{3}+\frac{t_{3}^{3} t_{4}^{2}}{5^{4}\left(t_{1}^{5}-t_{5}\right)}-2 t_{2} \tilde{t}_{7} \\
& \mathrm{~W}\left(\tilde{t}_{7}\right)=2 \tilde{t}_{7} \\
& \delta\left(\tilde{t}_{7}\right)=0 .
\end{aligned}
$$

Hence we get the vector fields $\mathrm{D}, \mathrm{W}$ and $\delta$ in the chart $\left(t_{1}, t_{2}, \ldots, t_{6}, \tilde{t}_{7}\right)$ as follows:

$$
\begin{aligned}
\mathrm{D} & =\left(t_{3}-t_{2} t_{1}\right) \frac{\partial}{\partial t_{1}}+\left(\frac{t_{3}^{3} t_{4}}{5^{4}\left(t_{1}^{5}-t_{5}\right)}-t_{2}^{2}\right) \frac{\partial}{\partial t_{2}} \\
& +\left(\frac{t_{3}^{3} t_{6}}{5^{4}\left(t_{1}^{5}-t_{5}\right)}-3 t_{2} t_{3}\right) \frac{\partial}{\partial t_{3}}-\tilde{t}_{7} \frac{\partial}{\partial t_{4}}-5 t_{2} t_{5} \frac{\partial}{\partial t_{5}} \\
& +\left(5^{5} t_{1}^{3}-2 t_{3} t_{4}-t_{2} t_{6}\right) \frac{\partial}{\partial t_{6}}+\left(-5^{4} t_{1} t_{3}+\frac{t_{3}^{3} t_{4}^{2}}{5^{4}\left(t_{1}^{5}-t_{5}\right)}-2 t_{2} \tilde{t}_{7}\right) \frac{\partial}{\partial \tilde{t}_{7}} \\
\mathrm{~W} & =t_{1} \frac{\partial}{\partial t_{1}}+2 t_{2} \frac{\partial}{\partial t_{2}}+3 t_{3} \frac{\partial}{\partial t_{3}}+5 t_{5} \frac{\partial}{\partial t_{5}}+t_{6} \frac{\partial}{\partial t_{6}}+2 \tilde{t}_{7} \frac{\partial}{\partial \tilde{t}_{7}} \\
\delta & =\frac{\partial}{\partial t_{2}}
\end{aligned}
$$

Indeed, here $t_{7} \notin \mathscr{M}^{2}{ }_{2}$, hence the space of $2 \mathrm{CY}$ modular forms is the following:

$$
\mathscr{M}^{2}=\mathbb{C}\left[t_{1}, t_{3}, t_{4}, t_{5}, t_{6}, \tilde{t}_{7}, \frac{1}{t_{5}\left(t_{5}-t_{1}^{5}\right)}\right]
$$

This transformation is new and it does not appear in pervious works.

Note that in [10] to solve the above problem we considered:

$$
\tilde{\mathrm{D}}:=\mathrm{D}-t_{2}\left(\left[\mathrm{D}, \frac{\partial}{\partial t_{2}}\right]-\mathrm{W}\right)=\mathrm{D}+t_{2} t_{4} \frac{\partial}{\partial t_{4}}-t_{2} t_{7} \frac{\partial}{\partial t_{7}}
$$


then we get:

$$
\begin{aligned}
\tilde{\mathrm{D}} & =\left(t_{3}-t_{1} t_{2}\right) \frac{\partial}{\partial t_{1}}+\left(\frac{t_{3}^{3} t_{4}}{5^{4}\left(t_{1}^{5}-t_{5}\right)}-t_{2}^{2}\right) \frac{\partial}{\partial t_{2}} \\
& +\left(\frac{t_{3}^{3} t_{6}}{5^{4}\left(t_{1}^{5}-t_{5}\right)}-3 t_{2} t_{3}\right) \frac{\partial}{\partial t_{3}}+\left(-t_{7}\right) \frac{\partial}{\partial t_{4}} \\
& +\left(-5 t_{2} t_{5}\right) \frac{\partial}{\partial t_{5}}+\left(5^{5} t_{1}^{3}-2 t_{3} t_{4}-t_{2} t_{6}\right) \frac{\partial}{\partial t_{6}}+\left(-5^{4} t_{1} t_{3}-2 t_{2} t_{7}\right) \frac{\partial}{\partial t_{7}}, \\
\tilde{\mathbf{W}} & =\mathbf{W}=t_{1} \frac{\partial}{\partial t_{1}}+2 t_{2} \frac{\partial}{\partial t_{2}}+3 t_{3} \frac{\partial}{\partial t_{3}}+5 t_{5} \frac{\partial}{\partial t_{5}}+t_{6} \frac{\partial}{\partial t_{6}}+2 t_{7} \frac{\partial}{\partial t_{7}}, \\
\tilde{\delta} & =\frac{\partial}{\partial t_{2}}
\end{aligned}
$$

which again form a copy of $\mathfrak{s l}_{2}(\mathbb{C})$. But we still could not find the $q$-expansion of a solution of $\tilde{D}$, which is not interesting. The Gauss-Manin connection matrix of $\tilde{D}$ is as follows:

$$
\left(\begin{array}{cccc}
0 & 1 & 0 & 0 \\
0 & 0 & \mathrm{Y}_{1} & 0 \\
t_{2} t_{4} & 0 & 0 & -1 \\
-t_{2}\left(t_{2} t_{4}+t_{7}\right) & t_{2} t_{4} & 0 & 0
\end{array}\right)
$$

\section{The case $n=4$}

In this case we get:

$$
\begin{aligned}
\mathrm{D} & =\left(t_{3}-t_{2} t_{1}\right) \frac{\partial}{\partial t_{1}}+\left(\frac{6^{-2} t_{3}^{2} t_{4} t_{8}}{t_{1}^{6}-t_{6}}-t_{2}^{2}\right) \frac{\partial}{\partial t_{2}} \\
& +\left(\frac{6^{-2} t_{3}^{2} t_{5} t_{8}}{t_{1}^{6}-t_{6}}-3 t_{2} t_{3}\right) \frac{\partial}{\partial t_{3}}+\left(\frac{-6^{-2} t_{3}^{2} t_{7} t_{8}}{t_{1}^{6}-t_{6}}-t_{2} t_{4}\right) \frac{\partial}{\partial t_{4}} \\
& +\left(\frac{6^{-2} t_{3} t_{5}^{2} t_{8}+5 t_{1}^{4} t_{3} t_{8}}{2\left(t_{1}^{6}-t_{6}\right)}-t_{3} t_{4}-2 t_{2} t_{5}\right) \frac{\partial}{\partial t_{5}} \\
& +\left(-6 t_{2} t_{6}\right) \frac{\partial}{\partial t_{6}}+\left(\frac{6^{-2} t_{4}^{2}-t_{1}^{2}}{2 \times 6^{-2}}\right) \frac{\partial}{\partial t_{7}}+\left(\frac{3 t_{1}^{5} t_{3} t_{8}}{t_{1}^{6}-t_{6}}-3 t_{2} t_{8}\right) \frac{\partial}{\partial t_{8}} \\
\mathrm{~W} & =t_{1} \frac{\partial}{\partial t_{1}}+2 t_{2} \frac{\partial}{\partial t_{2}}+3 t_{3} \frac{\partial}{\partial t_{3}}+t_{4} \frac{\partial}{\partial t_{4}}+2 t_{5} \frac{\partial}{\partial t_{5}}+6 t_{6} \frac{\partial}{\partial t_{6}}+3 t_{8} \frac{\partial}{\partial t_{8}}, \\
\delta & =\frac{\partial}{\partial t_{2}}
\end{aligned}
$$

where the equation $t_{8}^{2}=36\left(t_{1}^{6}-t_{6}\right)$ holds among $t_{i}$ 's. Analogous to the pervious cases we have $\operatorname{deg}\left(\mathrm{t}_{1}\right)=1, \operatorname{deg}\left(\mathrm{t}_{2}\right)=2, \operatorname{deg}\left(\mathrm{t}_{3}\right)=3, \operatorname{deg}\left(\mathrm{t}_{4}\right)=1, \operatorname{deg}\left(\mathrm{t}_{5}\right)=2, \operatorname{deg}\left(\mathrm{t}_{6}\right)=$ $6, \operatorname{deg}\left(\mathrm{t}_{7}\right)=0, \operatorname{deg}\left(\mathrm{t}_{8}\right)=3$. In this case also we can find the $q$-expansion of a solution components of $\mathrm{D}$ and their first 7 coefficients are given in Table 2. If we 
compute the $q$-expansion of $\mathrm{Y}_{1}^{2}$, then we find

$$
\begin{aligned}
\frac{1}{6} Y_{1}^{2} & =\frac{1}{6}\left(-Y_{2}\right)^{2}=\frac{1}{6^{3}} \frac{t_{3}^{4}}{t_{1}^{6}-t_{6}} \\
& =6+120960 q+4136832000 q^{2}+148146924602880 q^{3}+5420219848911544320 q^{4} \\
& +200623934537137119778560 q^{5}+\ldots
\end{aligned}
$$

which is the 4-point function discussed in [3, Table $1, d=4]$. We have also computed the $q$-expansion of the modular coordinate $z$

$$
\frac{z}{6^{6}}=\frac{t_{6}}{\left(6 t_{1}\right)^{6}}=q-6264 q^{2}-8627796 q^{3}-237290958144 q^{4}-4523787606611250 q^{5}+\cdots
$$

which coincides with the one computed in [4, §6.1].

\begin{tabular}{|c|c|c|c|c|c|c|c|}
\hline & $q^{0}$ & $q^{1}$ & $q^{2}$ & $q^{3}$ & $q^{4}$ & $q^{5}$ & $q^{6}$ \\
\hline$\frac{1}{20} t_{1}$ & $\frac{1}{720}$ & 1 & 4131 & 51734044 & 918902851011 & 19562918469120126 & 465569724397794578388 \\
\hline$\frac{1}{216} t_{2}$ & $-\frac{1}{216}$ & 9 & 110703 & 2248267748 & 55181044614231 & 1498877559908208054 & 43378802521495632926652 \\
\hline$\frac{1}{14} t_{3}$ & $-\frac{1}{504}$ & 11 & 115137 & 2265573692 & 54820079452449 & 1477052190387154386 & 42523861222488896739828 \\
\hline$\frac{1}{24} t_{4}$ & $-\frac{1}{144}$ & 16 & 193131 & 3904146832 & 95619949713765 & 2594164605185043648 & 75018247757143686903060 \\
\hline$\frac{1}{2} t_{5}$ & $-\frac{1}{144}$ & 45 & 469872 & 9215455916 & 222628516313454 & 5992746995783064438 & 172421735348939185816992 \\
\hline$-6^{6} t_{6}$ & 0 & -1 & 1944 & 10066356 & 139857401664 & 2615615263199250 & 57453864811412558112 \\
\hline$-\frac{1}{2} t_{7}$ & $-\frac{1}{72}$ & 7 & 32859 & 414746092 & 7395891627375 & 157811370338782458 & 3761184845284146266940 \\
\hline$\frac{18}{7} t_{8}$ & $-\frac{1}{3024}$ & 7 & 54855 & 1034706148 & 24546181658391 & 653902684588247058 & 18687787944102314534628 \\
\hline
\end{tabular}

Table 2: Coefficients of $q^{k}, 0 \leq k \leq 6$, in the q-expansion of a solution of $\mathrm{D}=\mathrm{D}_{4}$.

\section{Acknowledgments}

During a conversation with Don Zagier, he suggested to write a text without technical and computational details. So, the author would like to thank him for giving the idea of writing the present survey, which has been prepared during his stay at Max Planck Institute for Mathematics (MPIM) in Bonn. The author also expresses his gratitude to MPIM and its staff for preparing such an excellent ambience for doing mathematical work.

\section{References}

[1] Candelas, P.; de la Ossa, X. C.; Green, P. S.; Parkes, L.: A pair of CalabiYau manifolds as an exactly soluble superconformal theory, Nuclear Phys. B, 359(1):21-74, 1991.

[2] Cohen, H.: Sums involving the values at negative integers of L-functions of quadratic characters, Math. Ann., 217:81-94, 1977. 
[3] Greene, B. R.; Morrison, D. R.; Plesser, M. R.: Mirror manifolds in higher dimension, Comm. Math. Phys., 173(3):559-597, 1995.

[4] Klemm, A.; Pandharipande, R.: Enumerative geometry of Calabi-Yau 4-folds, Commun. Math. Phys., 281(3):621-653, 2008.

[5] Movasati, H.: Quasi modular forms attached to elliptic curves, I. Annales Mathématique Blaise Pascal, 19, 307-377, 2012.

[6] Movasati, H.: Modular-type functions attached to mirror quintic Calabi-Yau varieties, Math. Zeit., 281, Issue 3, pp. 907-929(3):907-929, 2015.

[7] Movasati, H.: Gauss-Manin connection in disguise: Calabi-Yau modular forms, International Press, Somerville, Massachusetts, U.S.A, and Higher Education Press, Beijing, China, 2017.

[8] Movasati, H.; Nikdelan, Y.: Gauss-Manin Connection in Disguise: DworkFamily, J. Differential Geometry, 119: 73-98, 2021.

[9] Nikdelan, Y.: Modular vector fields attached to Dwork family: $\mathfrak{s l}_{2}(\mathbb{C})$ Lie algebra, Moscow Math. J., 20(1):127-151, 2020.

[10] Nikdelan, Y.: Rankin-Cohen brackets for Calabi-Yau modular forms, arXiv:1912.12809 [math.NT], 2019.

[11] Nikdelan, Y.: Ramanujan-type systems of nonlinear ODEs for $\Gamma_{0}(2)$ and $\Gamma_{0}(3)$, arXiv:2111.01844 [math.NT], 2021.

[12] Zagier, D.: Modular forms and differential operators. Proceedings Mathematical Sciences, 104(1):57-75, 1994. 